Revista Arbitrada Interdisciplinaria de Ciencias de la Salud. SALUD Y VIDA

Volumen 3. Número 6. Año 3. Julio - Diciembre 2019

Hecho el depósito de Ley: FA2016000010

ISSN: $2610-8038$

FUNDACIÓN KOINONIA (F.K).

Santa Ana de Coro, Venezuela.

María Gabriela Bermúdez Moreira

http://dx.doi.org/10.35381/s.v.v3i6.358

\title{
Riesgos laborales ergonómicos en el área de distribución de una empresa del sector eléctrico
}

\section{Ergonomic occupational hazards in the distribution area of a company in the electricity sector}

\author{
María Gabriela Bermúdez Moreira \\ reddeinvestigacionrik@fundacionkoinonia.com.ve \\ Universidad Laica Eloy Alfaro de Manabí \\ Ecuador
}

Recibido: 1 de mayo de 2019

Aprobado: 31 de mayo de 2019

\begin{abstract}
RESUMEN
La investigación se basó en estudiar los Riesgos laborales ergonómicos en el área de distribución de una empresa del sector eléctrico, específicamente por los linieros. La metodología empleada fue de tipo exploratoria con la finalidad de conocer los riesgos ergonómicos del área de distribución de una empresa del sector eléctrico, se observa que los factores de riesgos ergonómicos provocan alteraciones de la salud, en el presente estudio vemos que las condiciones de trabajo del cargo de los linieros afectan a capacidad física del individuo, la cual disminuye después de haber realizado trabajos durante un determinado periodo de tiempo expuesto a; posturas forzadas, movimientos repetitivos, esfuerzo muscular localizado mantenido y esfuerzo físico general, mismos que al finalizar la larga jornada provocan que baje el ritmo de actividad del liniero disminuyendo el rendimiento en cantidad y calidad.
\end{abstract}

Descriptores: Empresa; Seguridad; Prevención de desastres.

\section{ABSTRACT}

The research was based on studying ergonomic occupational hazards in the distribution area of a company in the electricity sector, specifically by linemen. The methodology used was of exploratory type in order to know the ergonomic risks of the distribution area of a company in the electricity sector, it is observed that the ergonomic risk factors cause health alterations, in the present study we see that the working conditions The position of the linemen affects the physical capacity of the individual, which decreases after having performed work for a certain period of time exposed to; forced postures, repetitive movements, maintained localized muscular effort and general physical effort, 
Revista Arbitrada Interdisciplinaria de Ciencias de la Salud. SALUD Y VIDA

Volumen 3. Número 6. Año 3. Julio - Diciembre 2019

Hecho el depósito de Ley: FA2016000010

ISSN: $2610-8038$

FUNDACIÓN KOINONIA (F.K).

Santa Ana de Coro, Venezuela.

María Gabriela Bermúdez Moreira

which at the end of the long day cause the lineman's activity to decrease, reducing performance in quantity and quality.

Descriptors: Enterprises; Safety; Disaster prevention.

\section{INTRODUCCIÓN}

El recurso humano que se encarga del mantenimiento de líneas, redes, y circuitos eléctricos se lo denomina liniero electricista. El cargo es conocido en el mundo eléctrico como una de las labores que exige de gran esfuerzo físico, dado que por el tiempo en que se debe permanecer en posiciones forzosas, así como por el desarrollo de actividades que requieren de fortaleza y temples se le suman otra gran cantidad de factores a los que se ve expuesto el personal, el factor de riesgo eléctrico, el factor de riesgo de biomecánico por el trabajo en alturas y por las posiciones prolongadas, incluyendo los riesgos físicos por exposición a los rayos ultravioletas, riesgos químicos, biológicos y ergonómicos que hacen de esta actividad un cargo de mucho valor.

La Ley 31/1995, de 8 de noviembre, de Prevención de Riesgos Laborales es la norma legal por la que se determinan las garantías y responsabilidades precisas para establecer un adecuado nivel de protección de la salud de los trabajadores frente a los riesgos derivados de las condiciones de trabajo. De acuerdo a información preliminar en el tema de seguridad y salud ocupacional, se conoce que los linieros están frecuentemente expuestos a diferentes riesgos a lo largo de su jornada laboral, acumulando enfermedades por microorganismos, lesiones por fracturas, quemaduras, y hasta lesiones osteomusculares originadas por sus métodos de trabajo, aumentando así un sin número de afecciones que limita las funciones normales de su cuerpo comprometiendo su integridad física y mental.

Por este y muchos otros escenarios, para conseguir una protección adecuada para el trabajador la LPRL pone a disposición de las organizaciones cuatro materias preventivas: seguridad laboral, higiene industrial, ergonomía y psicosociología aplicada y la vigilancia de la salud, de manera que mediante la aplicación de estas disciplinas se logre la identificación oportuna de los riesgos, los posibles peligros derivados del uso de 
Revista Arbitrada Interdisciplinaria de Ciencias de la Salud. SALUD Y VIDA

Volumen 3. Número 6. Año 3. Julio - Diciembre 2019

Hecho el depósito de Ley: FA2016000010

ISSN: $2610-8038$

FUNDACIÓN KOINONIA (F.K).

Santa Ana de Coro, Venezuela.

María Gabriela Bermúdez Moreira

equipos, instalaciones, máquinas, útiles, sustancias, preparados y entornos laborales, se gestione una actuación preventiva apropiada, minimizando los accidentes que están relacionados con la actividad y los lugares de trabajo, así como las enfermedades profesionales.

La empresa eléctrica de Manabí se encarga de la comercialización y distribución de energía eléctrica en su área de concesión de aproximadamente 14,420m2; el personal del área de distribución se encarga tanto del mantenimiento y reparación de las redes eléctricas, así como de la instalación de las mismas dentro de este perímetro, por lo que los trabajadores de dicha área se exponen permanentemente a trabajar diferentes escenarios al aire libre, con condiciones variantes e insalubres y en muchas ocasiones sometidos a largas horas posiciones forzadas por la operación en los postes.

En la actualidad la empresa eléctrica cuenta con una acción preventiva sobre la que prevalece la seguridad laboral a función de prevenir los accidentes laborales, por lo que se trabaja sobre los riesgos de tipo mecánicos, ignorando totalmente la Higiene industrial y la ergonomía. Por esto, basados en la exposición, la probabilidad y las consecuencias a fin de mejorar las condiciones de salud de los linieros vemos la necesidad de enfatizar en el presente Trabajo de Fin de Master el análisis sobre los factores de riesgo ergonómicos, para de esta manera actuar sobre las condiciones de trabajo inseguras, socializando y concientizando a la clase obrera del área de distribución acerca de los mismos, fortaleciendo el sistema de prevención, disminuyendo los índices de lesiones, el ausentismo laboral, los costes para la empresa y el patrimonio de los colaboradores, mejorando su rendimiento y su concentración en el trabajo.

Conociendo esta problemática, de acuerdo a las características y actividades que se llevan a cabo en este entorno laboral, se considera necesario llevar a cabo un estudio cuyo problema de investigación se centra o gira alrededor de la siguiente pregunta particular ¿Cuáles son los riesgos ergonómicos a los que se tornan expuestos los trabajadores del área de distribución de la Empresa Eléctrica de la unidad de negocios 
Manabí? Con el objetivo de Conocer los riesgos ergonómicos del área de distribución de una empresa del sector eléctrico.

\section{Reseña de la empresa}

La Empresa eléctrica presta el servicio público de Distribución y Comercialización de energía eléctrica en varias regiones del Ecuador, se constituyó en sociedad anónima mediante escritura pública de fusión el 15 de diciembre de 2008; y, conformo disueltas empresas eléctricas de distribución en diferentes provincias del país entre esta la regional de Manabí.

La empresa eléctrica tiene como misión el brindar el servicio público de distribución y comercialización de energía eléctrica para generar bienestar a los consumidores y contribuir al desarrollo del país, consolidándose como una empresa líder en la prestación del servicio eléctrico en el Ecuador.

La Estructura Organizacional de la empresa eléctrica, está conformada por Gerencias Corporativas, y Unidades de Negocio cuya base se establece en base a las

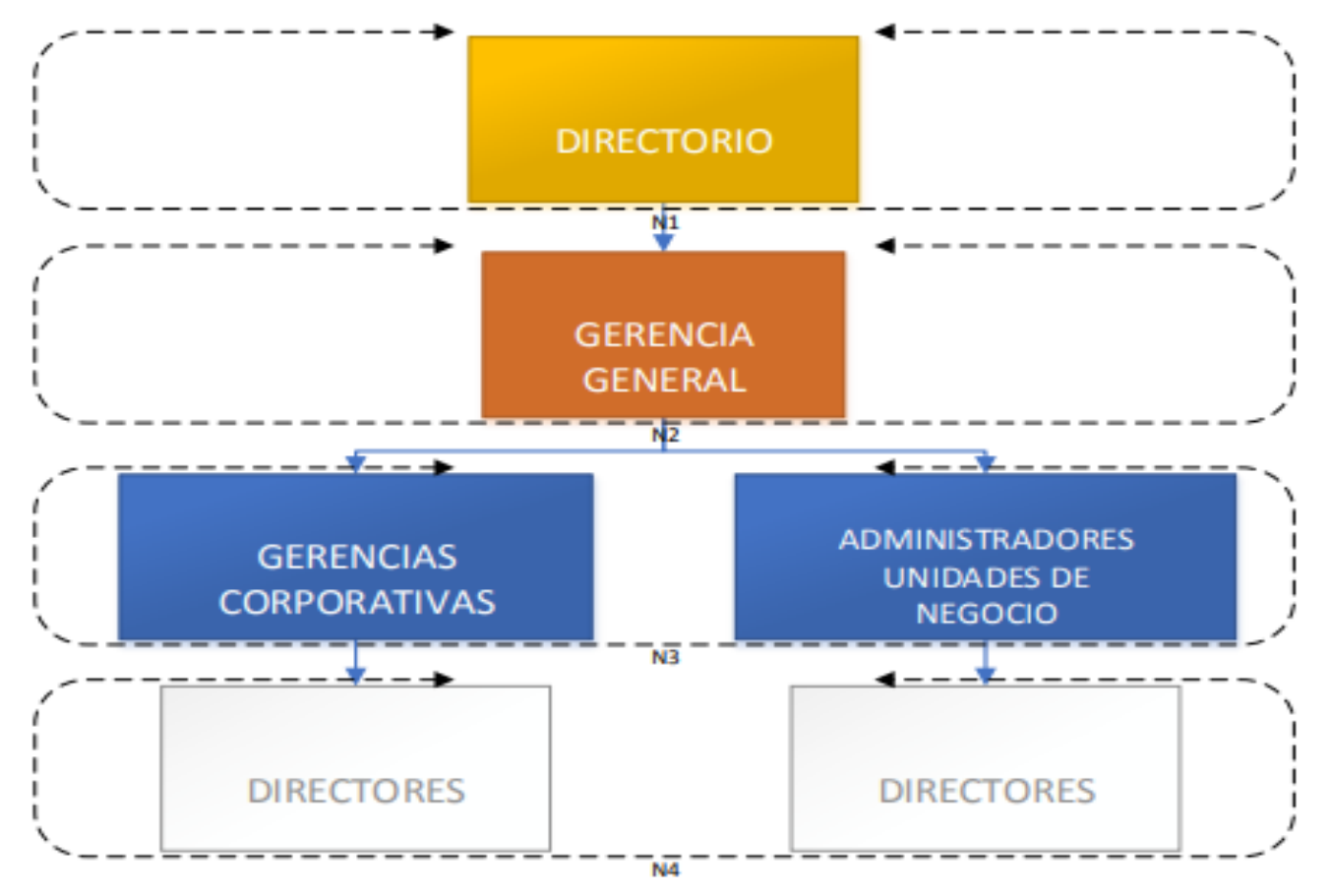

necesida des y condicion es de la Corporac ión, optimiza ndo los recursos disponibl es. 
Revista Arbitrada Interdisciplinaria de Ciencias de la Salud. SALUD Y VIDA

Volumen 3. Número 6. Año 3. Julio - Diciembre 2019

Hecho el depósito de Ley: FA2016000010

ISSN: 2610-8038

FUNDACIÓN KOINONIA (F.K)

Santa Ana de Coro, Venezuela.

María Gabriela Bermúdez Moreira

En las diferentes Unidades de negocio se trabaja bajo un mismo esquema organizacional así, se establecen líneas de autoridad a través de los diversos niveles y se delimitan las responsabilidades de cada cargo a lo largo de la cadena de valor. La empresa sobre la que se basa el presente estudio cuenta con un total de 756 empleados, de los cuales 380 son operativos 376 administrativos, cumpliendo un horario de labores de $8 \mathrm{hr}$ y guardias operativas con turnos rotativos en horarios de 24/7. El personal del área de distribución es el responsable de ejecutar la mantención de las redes de distribución eléctrica en baja y media tensión dentro de los estándares esperados del sector.

\section{METODOLOGÍA DE LA INVESTIGACIÓN}

La metodología empleada fue de tipo exploratoria con la finalidad de conocer los riesgos ergonómicos del área de distribución de una empresa del sector eléctrico, esto permitió en el caso específico de los linieros de la empresa, estudiar los riesgos ergonómicos a los cuales se ven sometidos. (Hernández Sampieri, Fernández Collado 
Revista Arbitrada Interdisciplinaria de Ciencias de la Salud. SALUD Y VIDA

Volumen 3. Número 6. Año 3. Julio - Diciembre 2019

Hecho el depósito de Ley: FA2016000010

ISSN: $2610-8038$

FUNDACIÓN KOINONIA (F.K).

Santa Ana de Coro, Venezuela.

María Gabriela Bermúdez Moreira

\& Baptista 2014), consideran que este tipo de investigación permite indagar un tema cuando este ha sido poco explorado o investigado, cimentando las primeras bases para proseguir en la profundización del conocimiento.

\section{RESULTADOS}

En el desarrollo del presente estudio se pudo evidenciar que no existe muchas investigaciones referentes a los problemas osteomusculares de los linieros, puesto que esta problemática ha sido poco estudiada, se identifican protocolos y guías para la realización de tareas específicas en otras condiciones de trabajo (sector del servicio, sector de la construcción, etc.), pero hay pocos estudios donde se enfatice en la mejora de la salud y condiciones de los linieros. Lo que supone caminos abiertos para futuras investigaciones sobre los efectos específicos que trae a la salud de las personas, desenvolverse en las condiciones en que se ostenta el cargo de liniero.

La empresa del sector eléctrico sobre la cual se realiza el presente estudio, no cuenta con un historial médico anterior a la información levantada hace 12 meses, por lo que no es posible verificar si las molestias patológicas (osteomuscular) son crónicas o agudas. En los resultados de la aplicación de los instrumentos de recolección de datos (cuestionario nórdico), se evidencian algunas sintomatologías músculo - esqueléticas presentadas en el cargo de liniero, por lo que es trascendente que se inicien actividades preventivas a fin de mejorar la calidad de vida de los colaboradores, minimizando la probabilidad de lesiones o enfermedades que pueden ser causa de la actividad laboral desarrollada en la empresa.

Es importante trabajar con los empleados en la formación acerca del reporte de incidentes o accidentes, ya que en la aplicación de los cuestionarios se evidencio que muchos colaboradores consideran normal las molestias osteomusculares presentadas por la ejecución del trabajo, durante o después de la jornada laboral.

Por otro lado es muy importante resaltar que implementar medidas preventivas basadas en la calentamiento física y estiramiento del cuerpo, acorde con lo publicado por la facultad del Deporte de la Universidad de Oporto, Portugal en su artículo "Efecto de un 
Revista Arbitrada Interdisciplinaria de Ciencias de la Salud. SALUD Y VIDA

Volumen 3. Número 6. Año 3. Julio - Diciembre 2019

Hecho el depósito de Ley: FA2016000010

ISSN: 2610-8038

FUNDACIÓN KOINONIA (F.K).

Santa Ana de Coro, Venezuela.

María Gabriela Bermúdez Moreira

programa", donde se detalla que luego de 6 meses de estudios se obtuvieron resultados significativos con respecto a la diminución de la intensidad del dolor en algunas de las regiones del cuerpo evaluados, gracias a la actividad física y al estiramiento previo de los músculos.

\section{CONCLUSIONES}

En el análisis de las actividades desarrolladas por el personal, se observa que los factores de riesgos ergonómicos provocan alteraciones de la salud, en el presente estudio vemos que las condiciones de trabajo del cargo de los linieros afectan a capacidad física del individuo, la cual disminuye después de haber realizado trabajos durante un determinado periodo de tiempo expuesto a; posturas forzadas, movimientos repetitivos, esfuerzo muscular localizado mantenido y esfuerzo físico general, mismos que al finalizar la larga jornada provocan que baje el ritmo de actividad del liniero disminuyendo el rendimiento en cantidad y calidad, así como también que los movimientos del personal se hagan más torpes e inseguros, por ello es necesario que se logre una adaptación y mejora de las condiciones de trabajo para los linieros.

En la actualidad tanto las empresas públicas y privadas están comprometidas en mejorar la calidad de vida de sus colaboradores, por cuanto se considera que la fuerza obrera es un eslabón importante en la cadena de producción, por lo que se hace cada vez más necesario invertir en el capital humano. Enfocados en este precepto la empresa eléctrica deberá realizar una importante inversión en los procesos de salud ocupacional, realizando una vigilancia de la salud apropiada que permita identificar fallos en las condiciones de trabajo, en el cumplimiento de los procedimientos y en los planes de prevención realizados.

Entre una de las medidas preventivas encaminadas a la disminución de las patologías derivadas del riesgo ergonómico se sugiere la inversión para sustituir los "cinturones de seguridad" por "arneses de cuerpo completo", para lograr evidenciar si con esta medida se disminuyen las molestias a nivel de la columna, las cuales son las más frecuentes en el cargo de liniero según el cuestionario aplicado. 
Es importante acotar que también es primordial que se le haga conocer a los trabajadores los factores de riesgo a los que están expuestos en su lugar de trabajo, a juicio de Aldana, Isea, Ramos \& Martínez (2019), esto permite establecer un clima organizacional favorable, así mismo que siempre deben estar alerta a todos los procesos y cambios en su salud, notificando los mismos y que esto no va a ser sujeto de observación o recriminación en su lugar de trabajo, ya que con la información brindada de ser necesario se analizaran e implantaran mejoras en su espacio laboral, aunado a lo planteado por Ramírez Sánchez \& Franco (2019), plantean que los servidores públicos deben tener un compromiso organizacional para minimizar los factores de riesgo en la empresa. Para esto es importante hacer conocer y practicar las siguientes recomendaciones con el propósito de que el personal actué en pro de su bienestar físico:

- Organizar el trabajo considerando la alternancia entre las tareas, y el ritmo de trabajo.

- Realizar pausas en el trabajo para cambiar de postura y cambiar de postura periódicamente, si el esfuerzo requiere movimientos excesivamente repetitivos.

- Formar e informar a los trabajadores sobre los métodos de trabajo que reduzcan el riesgo de lesiones osteomusculares.

- Respetar los límites de peso manipulado, y utilizar unas técnicas adecuadas en el manejo de cargas si se va a manipular la carga manualmente.

- Fomentar hábitos posturales correctos en el trabajo, así como ejercicios de autocuidado.

- Establecer medidas organizativas como, por ejemplo, la rotación de puestos de trabajo si la tarea a realizar es demasiado pesada.

- Realizar la vigilancia de la salud de acuerdo con los protocolos específicos por exposición a riesgos del trabajo.

- Efectuar reconocimientos médicos periódicos que faciliten la detección de posibles lesiones musculo-esqueléticas. 


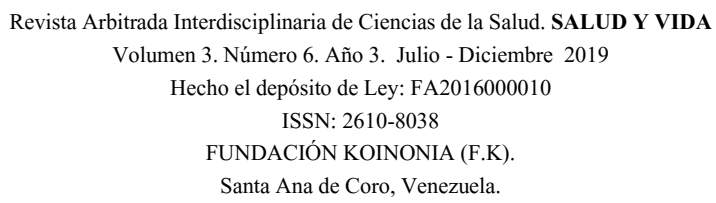

María Gabriela Bermúdez Moreira

- Supervisar los métodos de manipulación, manejar cargas pesadas entre dos o más personas y sustituir la manipulación manual, por mecánica, en la medida que sea posible.

\section{REFERENCIAS CONSULTADAS}

1. Aldana, J., Isea, J., Ramos, J. y Martínez, A. (2019). Análisis de la estructura organizacional basada en centros integrales de servicios sobre el clima organizacional de Corpoelec Falcón, Venezuela. Emprende y Transforma, 1(1), 71-79. https://doi.org/10.33829/emprendeytransforma-0101-2019-71-79

2. Ergonomia. (2003). Sociedad de Ergonomía y factores humanos de México. Retrieved from http://alebrige. uam.mx/ergonomía/ergouam.htl.

3. Ergonomia.cl. (2014, MAYO 18). Cuestionario Nórdico de Kuorinka ergonomia.cl.

4. Retrieved from Cuestionario Nórdico de Kuorinka - ergonomia.cl: http://www.ergonomia.cl/eee/Inicio/Entradas/2014/5/18 Cuestionario Nordico de Kuorinka.html

5. Gardey, J. P. (2013). Definición de. Retrieved from Definición de.: https://definicion.de/salud-ocupacional//

6. Gardey., J. P. (2013). Definicion.de. Retrieved from Definicion.de: https://definicion.de/prevencion-de-riesgos/)

7. Hernández Sampieri, R., Fernández Collado , C., \& Baptista , L. (2014).

8. Metodología de la investigación . México: Editorial Mc - Graw - Hill Interamericana. Sexta edición.

9. Ramírez Sánchez, V., \& Franco, F. (2019). Evaluación del Compromiso

10. Organizacional de Servidores Públicos en México. Revista Arbitrada Interdisciplinaria Koinonía, doi:http://dx.doi.org/10.35381/r.k.v4i8.263 
Revista Arbitrada Interdisciplinaria de Ciencias de la Salud. SALUD Y VIDA

Volumen 3. Número 6. Año 3. Julio - Diciembre 2019

Hecho el depósito de Ley: FA2016000010

ISSN: 2610-8038

FUNDACIÓN KOINONIA (F.K).

Santa Ana de Coro, Venezuela.

María Gabriela Bermúdez Moreira

\section{REFERENCES CONSULTED}

1. Aldana, J., Isea, J., Ramos, J. and Martínez, A. (2019). Analysis of the organizational structure based on comprehensive service centers on the organizational climate of Corpoelec Falcón, Venezuela. Undertake and Transform, 1 (1), 71-79. https://doi.org/10.33829/emprendeytransforma-0101$\underline{2019-71-79}$

2. Ergonomics. (2003). Ergonomics and human factors society of Mexico. Retrieved from http://alebrige.uam.mx/ergonomía/ergouam.htl

3. Ergonomics.cl (2014, MAY 18). Nordic Questionnaire of Kuorinka - ergonomia.cl.

4. Retrieved from Kuorinka Nordic Questionnaire - ergonomia.cl: http://www.ergonomia.cl/eee/Inicio/Entradas/2014/5/18 Korinka Nordico Questi onario html

5. Gardey, J. P. (2013). Definition of. Retrieved from Definition of: https://definicion.de/salud-ocupacional/

6. Gardey., J. P. (2013). Definition of. Retrieved from Definicion.de: https://definicion.de/prevencion-de-riesgos/)

7. Hernández Sampieri, R., Fernández Collado, C., \& Baptista, L. (2014).

8. Investigation methodology . Mexico: Editorial Mc - Graw - Interamerican Hill. Sixth edition.

9. Ramírez Sánchez, V., \& Franco, F. (2019). Evaluación del Compromiso

10. Organizacional de Servidores Públicos en México. Revista Arbitrada Interdisciplinaria Koinonía, 4(8), 166-189. doi:http://dx.doi.org/10.35381/r.k.v4i8.263 